\title{
Fabrication and Investigation of Spectral Properties of a Dielectric Slab Waveguide Photonic Crystal Based Fano-Filter
}

\author{
Yousuf Khan ${ }^{1,2, *}$, Atiq Ur Rehman ${ }^{2}\left({ }^{\oplus}\right.$, Bibi A. Batool ${ }^{2}$, Mahain Noor ${ }^{2}$, Muhammad A. Butt ${ }^{3,4}(\mathbb{D}$, \\ Nikolay L. Kazanskiy 4,5 (D) and Svetlana N. Khonina ${ }^{4,5}$ \\ 1 Institute of Nanostructure Technologies and Analytics, University of Kassel, Heinrich-Plett-Str. 40, \\ 34132 Kassel, Germany \\ 2 Department of Electronic Engineering, Balochistan University of Information Technology, Engineering and \\ Management Sciences, Quetta 87300, Pakistan; atiqkhantareen@gmail.com (A.U.R.); \\ syedaasma142@gmail.com (B.A.B.); mahain.baloch.923@gmail.com (M.N.) \\ 3 Warsaw University of Technology, Institute of Microelectronics and Optoelectronics, Koszykowa 75, \\ 00-6625 Warszawa, Poland; butt.m@ssau.ru \\ 4 Samara National Research University, 443086 Samara, Russia; kazanskiy@ipsiras.ru (N.L.K.); \\ khonina@ipsiras.ru (S.N.K.) \\ 5 Image Processing Systems Institute, Russian Academy of Sciences, 443001 Samara, Russia \\ * Correspondence: yousuf.naudhani@gmail.com
}

\section{Citation: Khan, Y.; Rehman, A.U.} Batool, B.A.; Noor, M.; Butt, M.A.; Kazanskiy, N.L.; Khonina, S.N.

Fabrication and Investigation of Spectral Properties of a Dielectric Slab Waveguide Photonic Crystal Based Fano-Filter. Crystals 2022, 12, 226. https://doi.org/10.3390/ cryst12020226

Academic Editors:

Alessandro Chiasera and

George Kenanakis

Received: 17 December 2021

Accepted: 3 February 2022

Published: 5 February 2022

Publisher's Note: MDPI stays neutral with regard to jurisdictional claims in published maps and institutional affiliations.

Copyright: (C) 2022 by the authors. Licensee MDPI, Basel, Switzerland. This article is an open access article distributed under the terms and conditions of the Creative Commons Attribution (CC BY) license (https:// creativecommons.org/licenses/by/ $4.0 /)$.

\begin{abstract}
In this paper, we discuss the fabrication of a dielectric photonic crystal (PhC)-based Fanofilter device, as well as a numerical investigation of its spectral characteristics. The process parameters affecting the structural and physical properties of the fabricated device are discussed in detail, along with their influence on the spectral properties of the filter. The considered experimental problem is a three-layered PhC structure, fabricated using focused ion-beam (FIB) technology, designed to operate in the near-infrared (NIR) range. The studied parameters include the shape of PhC elements, depth of the structures, cladding layer thicknesses, and the refractive index of the material. Numerical design and simulations are performed using an open-source python-based Finite-difference timedomain (FDTD) simulation tool. The proposed optical filter device operates based on the principle of guided-mode resonance, and achieved a maximum quality factor value in the range of 800 .
\end{abstract}

Keywords: dielectric photonic crystals; Fano-filter; focused ion-beam technology; structural profile; guided-mode resonance

\section{Introduction}

Photonic crystals (PhCs) are nanostructures capable of manipulating light at the wavelength scale. The idea of PhCs was first proposed by John [1] and Yablonovitch [2] in 1987, and they were suggested for application as optical filters by Magnessian and Wang [3] in the early 1990s. Since their invention, PhC structures have been suggested for various applications, including optical filters [4,5], waveguiding [6], metasurfaces [7], optical switches [8], logic gates [9], biomedical sensors [10], and in numerous photonic devices [11]. With the increasing demand for PhCs, their rapid prototyping and testing have become more essential. Commonly used fabrication techniques for $\mathrm{PhC}$ structures include electronbeam (E-beam) lithography, reactive-ion etching (RIE) [12], FIB milling lithography [13], nano-imprint lithography $[14,15]$, and material processing using high-energy femtosecond laser pulses [16]. However, achieving nanometre precision for feature size in the sub- $\mu \mathrm{m}$ range with high resolution and aspect ratio is still challenging when using conventional fabrication technologies. The structural profile and physical properties of the fabricated nanostructures may deviate from their numerically designed models, due to various process- and material-related parameters. These deviations become more pronounced when working with PhCs, which operate on the principle of Guided-mode Resonance (GMR), 
also known as Fano-resonance [17-27], which is very sensitive to the structural properties of the periodic structures. GMR works on the principle of out-of-plane coupling of light in the structures, where the free space modes interfere with leaky modes and guided modes inside the structures with a phase-matching mechanism to create resonances. At present, FIB is considered one of the most precise and rapid prototyping technologies for nanostructures such as $\mathrm{PhCs}$, as it offers many tuneable parameters, thus making the process very versatile. Despite its many advantages, however, FIB-based material processing also faces some challenges, such as surface charging, unnecessary corrosion of specimen surfaces, and long process durations. Due to their non-conductive properties and hardness, these effects become more significant when prototyping dielectric materials. To accelerate the process, high ion-beam currents can be used, which may reduce the quality of patterned structures. High beam currents can also result in other malfunctions, such as the re-deposition of milled materials, which may affect the physical properties of the fabricated structures. Besides other properties of fabricated structures, the refractive indices of the deposited materials may vary from the desired values, due to the process parameters and equipment calibration. An investigation of the effects of FIB process parameters on the properties of fabricated $\mathrm{PhCs}$ has been reported in $[13,28]$. The fabrication of nanostructures using direct patterning ion-beam lithography has been presented in [29]. Moreover, [30] has reported the use of FIB technology for the fabrication of microsystems, along with a discussion of related process parameters. The GMR phenomenon in dielectric photonic crystals has been studied in $[13,17,22,25,31]$. Some works have reported on the study of spectral responses of PhC structures, based on their structural profile, including $[13,17,19,23]$. Detailed studies of Fano-resonances in nanostructures have been reported in [17-27]. The various applications of dielectric PhCs have been reported in [32].

The focus of this work is to study the structural features of the FIB-fabricated PhC structures, as well as analyse the process parameters influencing them. To investigate the spectral characteristics and quality of the fabricated Fano-filter devices, their structural features are re-modelled using simulation software. The structural and physical properties of the numerical models are varied, as per the experimental results, and their effects on Fano-resonances are investigated in terms of the Quality Factor (QF), Linewidth, and shifting of resonant wavelengths $\lambda_{\text {res }}$. The proposed work is divided into four case studies, in which the shape of the PhC elements is studied, as well as their depth, the thickness of the cladding layer, and the refractive index of the waveguiding layer. The significance of this work lies in optimizing the fabrication parameters to obtain an optimal structural profile of $\mathrm{PhC}$, as well as addressing the challenges related to the optical characterization of dielectric Fano-filter devices.

\section{Materials and Methods}

To make the design easy to fabricate and mechanically stable, an all-solid three-layered dielectric slab waveguide structure was considered for fabrication, as shown in Figure 1. The fabrication of the discussed slab waveguide PhC device includes two main steps: Deposition of the thin-films and structuring the $\mathrm{PhC}$ elements. A step-by-step visualization of the fabrication procedure is depicted in Figure 1. In the first step, the dielectric thinfilms are deposited on a borosilicate glass substrate, using Ion-beam Sputter Deposition (IBSD) methods (Figure 1, steps 1-4). IBSD offers a good material quality of the deposited

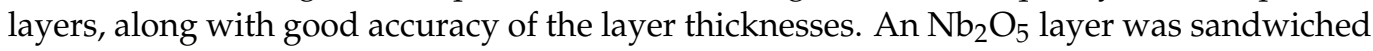
between two $\mathrm{SiO}_{2}$ cladding layers, to form a symmetric slab waveguide design. The $\mathrm{SiO}_{2}$ layers have thicknesses of $300 \mathrm{~nm}$ each, while the $\mathrm{Nb}_{2} \mathrm{O}_{5}$ is $330 \mathrm{~nm}$ thick. These design values were chosen as per the optimized theoretical model. The measured refractive index of the $\mathrm{Nb}_{2} \mathrm{O}_{5}$ layer in the NIR spectral range is $n_{2}=2.2$ and the $\mathrm{SiO}_{2}$ layers have a refractive index of $n_{1}=1.5[13,16]$. The fabrication-related dispersion properties of the considered materials are discussed in detail in [33]. To avoid an accumulation of surface charge on the surface of the dielectric material during FIB milling and scanning electron microscopy (SEM) imaging, the surface of the specimen was coated with a $10 \mathrm{~nm}$ thick 
platinum (Pt) layer (Figure 1, step 5). Moreover, the surface of the specimen was grounded with the stage of the specimen holder, in order to improve the accuracy of the process. A soft mask of the optimized model was designed in the Raith ELPHY [30] provided GDSII-compatible software platform (Figure 1, step 6). In the designed mask, the lattice constant of the periodic structure was kept at $a=1 \mu \mathrm{m}$ with a hole radius of $300 \mathrm{~nm}$. A direct patterning method offered by FIB technology was used, where the deflection of the ion beam was controlled following the designed soft mask, thus enabling rapid prototyping and reduced the complexity of the process. This process is also known as milling lithography. A live-milling sub-routine was used, where the FIB system enables SEM visualization of the fabrication process. In the final step of fabrication, the Pt layer was removed (Figure 1, step 8) by wet etching, as it can disturb the incident light beam during optical characterization of the device.

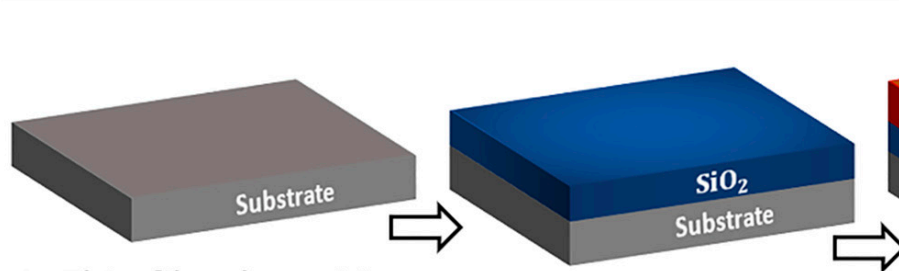

1. Thin film deposition using IBSD

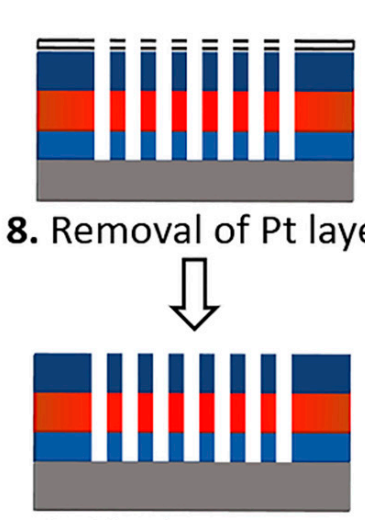

9. Final structure
2. $\mathrm{SiO}_{2}$ layer deposition

3. $\mathrm{Nb}_{2} \mathrm{O}_{5}$ layer deposition

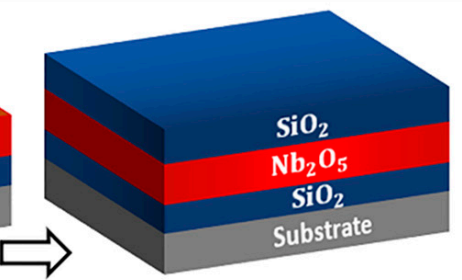

4. Deposition of another $\mathrm{SiO}_{2}$ layer

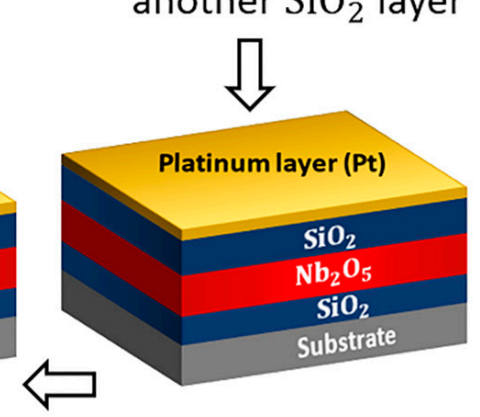

6. FIB milling lithography

Figure 1. Stepwise concluded fabrication of the dielectric PhC based Fano-filter.

SEM images of the fabricated structures are shown in Figure 2. The top and crosssectional views of the milled holes, with a depth of around $930 \mathrm{~nm}$, can be seen in Figure 2a, whereas SEM images of PhC elements with a depth of around $1.5 \mu \mathrm{m}$ and a conical structural profile can be seen in Figure $2 \mathrm{~b}$. The resultant structures are meant to be as close to the numerically optimized model as possible, where the milled holes ideally extend equally on both sides of the waveguide layer, having a depth of $930 \mathrm{~nm}$ (Figure 1, step 7). However, taking into consideration the material properties of the subject specimen and the FIB process parameters used, the structural properties of the fabricated structures deviated from the optimized theoretical model. The physical properties of the deposited thin-films, such as the refractive index, may also vary from the optimized value during the deposition process, due to the machine parameters and conditions of the vacuum chamber. The main FIB parameters influencing the structural properties of the milled holes include the ion-beam current, area dose, and number of process loops used. A low ion-beam current (e.g., $80 \mathrm{pA}$ ) can be used for high-quality milled structures or for creating cross-sectional views of nanostructures; however, this is not suitable for the fabrication of large-area structures, as the process duration would extend exponentially. Therefore, an ion-beam current in the range of $300 \mathrm{pA}$ to $1.5 \mathrm{nA}$ was used to pattern the $\mathrm{PhC}$ structures. 
However, the milled holes using these ion-beam currents tend to have a conical structural profile, rather than a standard cylindrical shape (Figure 2). Moreover, as the area dose is increased, the surface roughness of produced structures also increases. Additionally, if the number of process loops is not tightly controlled, the milled holes may become deeper than desired, as depicted in Figure 2b.
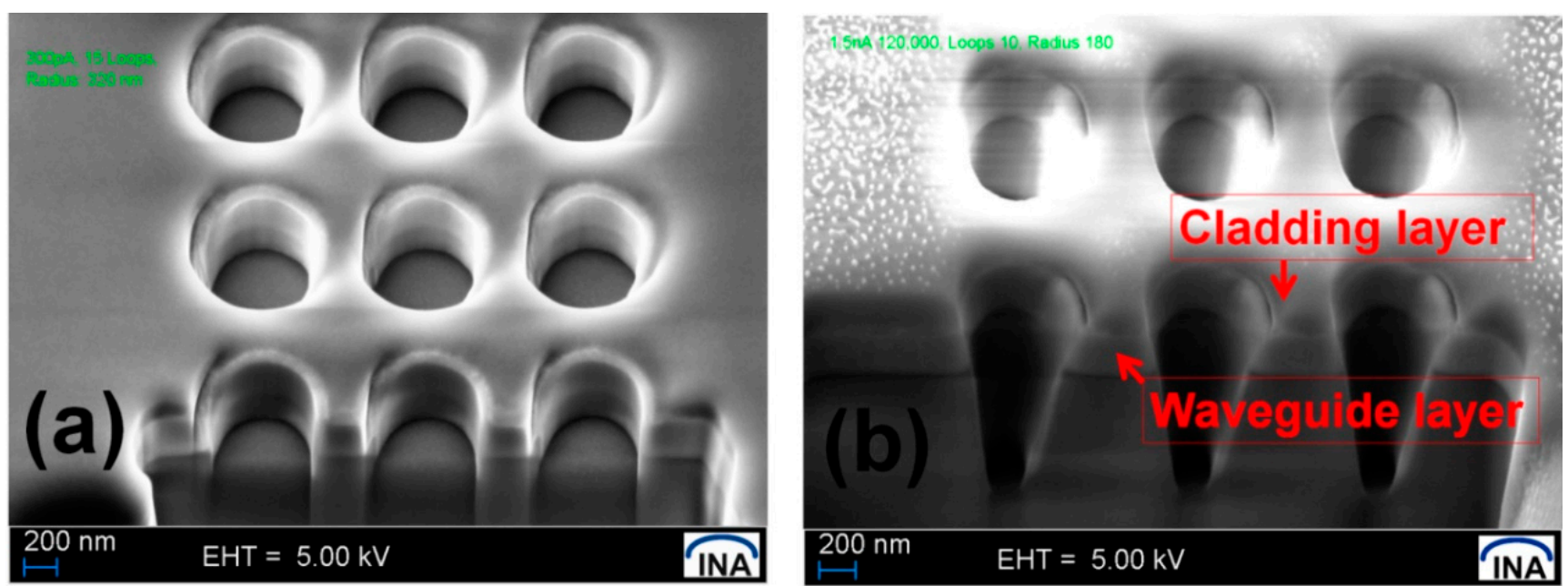

Figure 2. SEM images with a top and cross-sectional view of dielectric PhC structures fabricated using FIB milling lithography. (a) PhC-elements with a depth of $930 \mathrm{~nm}$ and air-holes extending equally on both sides of the waveguide. (b) Deep PhC-elements with conical structural profile and a depth of $1.5 \mu \mathrm{m}$.

\section{Simulation Model}

The numerical design and simulation of the proposed optical Fano-filter device were carried out using an open-source python-based FDTD simulation tool, known as MIT Electromagnetic Equation Propagation (MEEP) [34,35]. To investigate the effect of each structural shape on GMR and the resulting spectra in detail, a single-cell modelling technique was used. The single modelling approach can save computational resources and time, allowing the user to perform several simulations when studying a problem. For visual clarity, a 3D diagram of the PhC structure, along with an indicated excitation source on the top and lattice constant $a$, is shown in Figure 3a. The single-cell model (Figure 3b) was simulated as an infinite crystal through the application of Periodic Boundary Conditions (PBCs) in the lateral dimensions $(x$ and $y$ ). Figure $3 c$ depicts the cross-sectional view of the single-cell model, indicating the Perfectly Matched Layer (PML) boundary condition at the upper and lower boundaries (z-direction) of the model. The model shows a standard $\mathrm{PhC}$ element with vertical sidewalls and an air hole extending above and below the waveguide layer. The lattice constant of this model is $a=1$, meant for optical filters working in the NIR spectral range. The rest of the design parameters are expressed in terms of the lattice constant (e.g., the thickness of the waveguide is $0.33 a$ ), which enables the model to be scaled to different spectral ranges. A plane wave excitation source, a reflection monitor layer above the structure, a transmission monitor layer, and a decay monitor point are indicated in the model depicted in Figure 3c. The source is defined with Ex component at an incidence angle of 0 . As the discussed $\mathrm{PhC}$ structures have circular symmetry, there is no effect of source polarization on the results. The decay monitor point decides when to terminate the simulation, with respect to a user-defined source decay value. Moreover, the upper hole-radius $R_{t}$, bottom hole-radius $R_{b}$, depth of the air-hole $d$, and thickness of the cladding layer $c$ can be seen in the model. The default values of these design parameters were $R_{t} / R_{b}=0.30 / 0.30 a, d=0.33 a$, and $c=0.30 a$. After termination of the time-domain simulation, the recorded results are converted into the frequency domain for graphical visualization. 


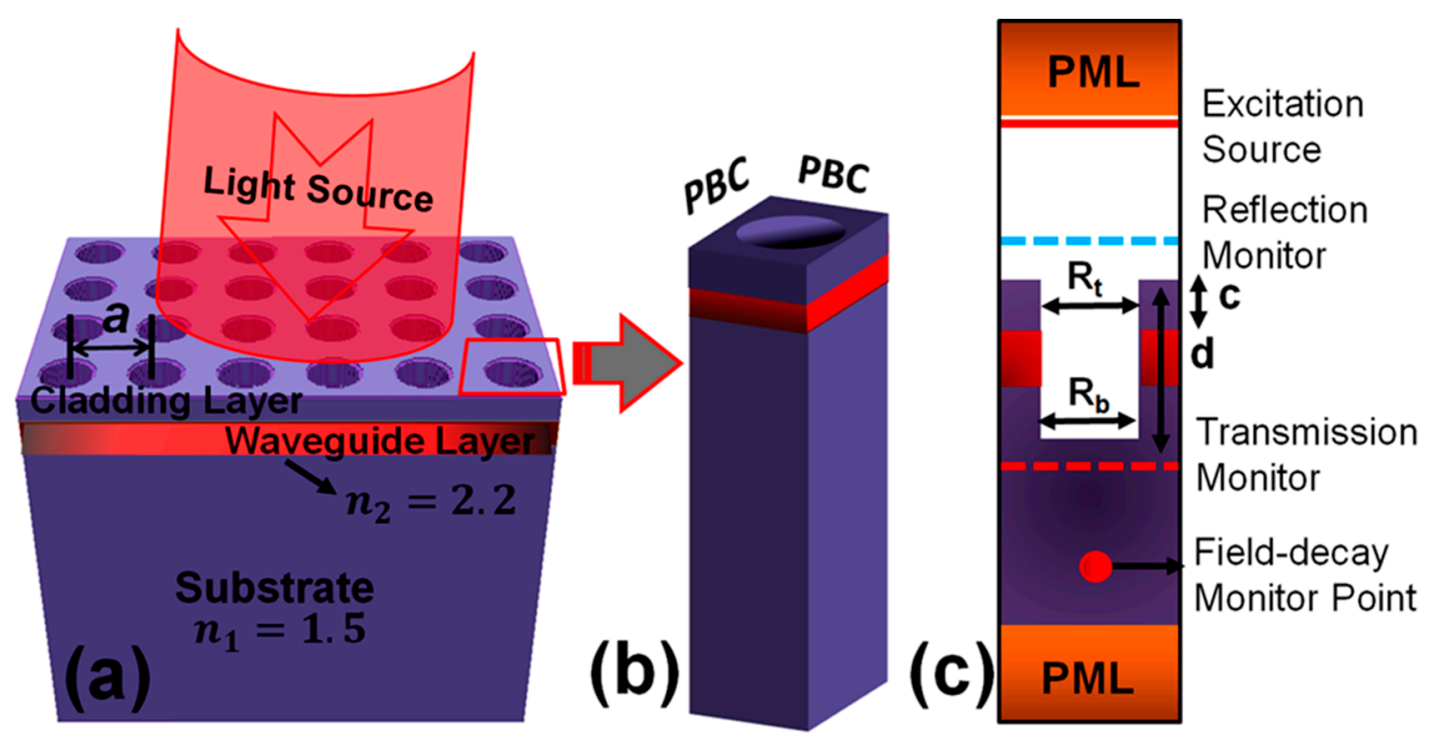

Figure 3. Simulation model of the proposed Fano-filter device. (a) 3D model of the slab waveguide PhC Fano-filter device with an indication of the excitation source. (b) Single-cell model with application of PBCs in lateral directions. (c) Cross-sectional view of the simulation model with excitation source, field monitor layers and field-decay monitor point.

\section{Spectral Characteristics of Fano-Filters}

It is well-known that the response of a Fano-filter is sensitive to the structural properties of the periodic structures and the spectral properties of the excitation source $[13,17,23,25,27]$. To study the influences of the structural and physical properties of the fabricated filter device on its spectral characteristics, the shape, depth, and thickness of the cladding layer of the $\mathrm{PhC}$ element were varied in the numerical models. Moreover, the refractive index of the waveguide was also varied above and below the optimum value, in order to study the influence of physical properties on the resonant modes. The linewidth and Quality Factor (QF) of the computed resonant modes were evaluated by fitting, using the Fano resonance lineshape function given by Equation (1):

$$
F(\omega)=A_{o}+F_{o} \frac{\left[q+2\left(\omega-\omega_{o}\right) / \Gamma\right]^{2}}{1+\left[2\left(\omega-\omega_{o}\right) / \Gamma\right]^{2}},
$$

where $\omega_{0}$ is the central frequency of the resonant mode, $\Gamma$ is the resonance linewidth, and $A_{o}$ and $F_{o}$ are constant values. Thus, the $\mathrm{QF}$ of the resonant modes is given by Equation (2):

$$
Q F=\frac{\lambda_{\text {res }}}{\Gamma} .
$$

\subsection{PhC Element Shape-Dependent Resonances}

After carefully observing the cross-sectional view of the fabricated structures (SEM images in Figure 2), numerical models were designed to investigate the effect of $\mathrm{PhC}$ element shape on the performance of the designed Fano-filter. The transmission spectrum of a standard PhC filter model with cylindrical holes is shown in Figure 4a. The spectra have two resonant modes, in the range of $\lambda_{\text {res }}=1340 \mathrm{~nm}$ and $1492 \mathrm{~nm}$. The PhC element shape was studied at an optimized hole-depth of $d=0.93 a$, where the air-holes extend equally on both sides of the waveguide. Keeping all the other design parameters constant, the shape of the $\mathrm{PhC}$ element was varied by increasing the top radius $R_{t}$ and decreasing the bottom radius $R_{b}$ in five steps, with a factor of $+0.05 a$ and $-0.05 a$, respectively, as shown in Figure $5 \mathrm{~d}$. The shapes of the PhC elements were changed in such a way that the radius at the centre of the waveguide remained constant at $0.30 a$. It can be seen, from the 
transmission spectra plot (Figure $4 \mathrm{~b}$ ), that both resonant modes observed a red-shift as the shape of the hole became more conical. It is important to mention here that the resonant mode 1 , around the $1500 \mathrm{~nm}$ wavelength range (also shown in the inset), was considered in the spectral analysis, as it had more pronounced peaks.
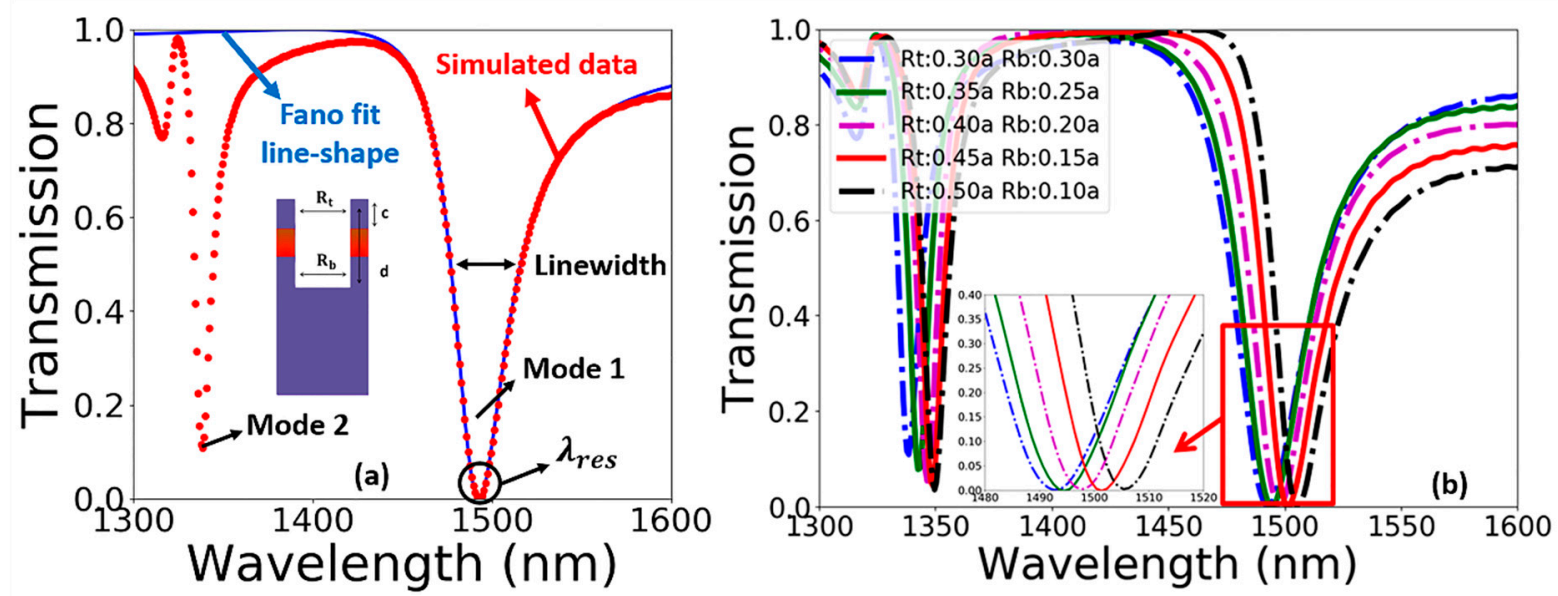

Figure 4. (a) Simulated (red) and Fano fit (blue) transmission spectrums of the filter with standard PhC-element of cylindrical holes $R_{t}=R_{b}=0.30 a$. (b) Transmission spectra of the filter as a function of wavelength with variation in the structural profile of $\mathrm{PhC}$-elements from cylindrical to conical shape.
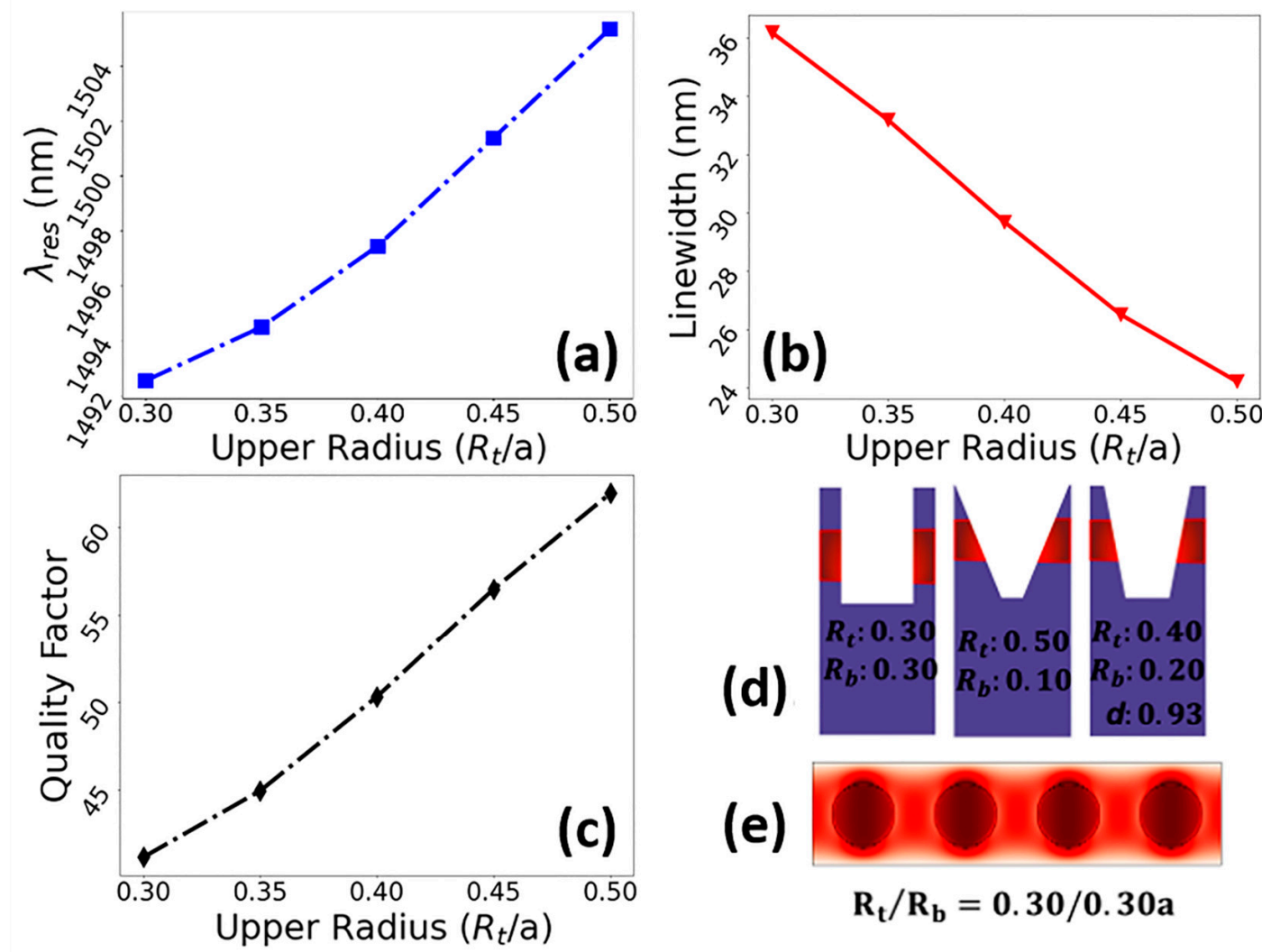

(e)

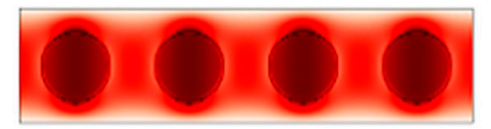

$$
\mathbf{R}_{\mathrm{t}} / \mathbf{R}_{\mathbf{b}}=0.30 / 0.30 \mathrm{a}
$$

Figure 5. Spectral characteristics of the Fano-filter device for variation in the shape of the PhCelements. (a) $\lambda_{\text {res }}$ as a function of $R_{t} / R_{b}$. (b) Linewidth as a function of $R_{t} / R_{b}$. (c) QF as a function of $R_{t} / R_{b}$. (d) Different shapes of PhC-elements used in simulation. (e) EM field distribution for $R_{t} / R_{b}=0.30 / 0.30 a$. 
A detailed graphical analysis of the spectral properties of the device, with respect to variation in the hole shape, is shown in Figure 5. The lines connecting the plotted simulation data are just guides to the eyes. The plot for $\lambda_{\text {res }}$ vs. $R_{t} / R_{b}$ in Figure 5 a shows that the resonant peaks observed a slightly exponential red-shift as the hole shape varied. The reason for this effect can be explained by a decrease or increase in the effective refractive index, $n_{\text {eff }}$ [22], of the periodic structure above and below the waveguide as the hole shape changes. It can also be concluded, from the plot, that the separation of the modes also increased as the $n_{\text {eff }}$ of the periodic structures increased. It can be observed that the linewidth in Figure 5b decreased and the QF in Figure 5c increased as the hole shape transformed to conical. It can be observed that, at a PhC element shape value of $R_{t} / R_{b}=0.50 / 0.10 a$, the linewidth reached its lowest value (around $25 \mathrm{~nm}$ ) and the QF achieved its maximum value (around 62). Moreover, the EM field distribution diagram in Figure 5e shows the localization of resonant modes inside the PhC elements during the occurrence of GMR.

\subsection{PhC Element Depth Dependence of Resonances}

During fabrication of the filter device, the FIB milling routine is divided into multiple process loops, in order to control the depth of the structures through the number of added process loops. However, when using high beam currents, such as $300 \mathrm{pA}$ and $1.5 \mathrm{nA}$, it is very challenging to control the depth of the milled holes in the $\mathrm{nm}$ range. Therefore, it is crucial to investigate the effect of hole depth on the spectral properties of the proposed Fanofilter device. As the structures resulting from FIB milling bear a conical shape, an optimized experimental model with $R_{t} / R_{b}=0.40 / 0.20 a$ was used in the simulations, in order to investigate the spectral features of the device (Figure 6a). The transmission spectra with variation in the depth of the PhC elements from $d=0.30 a$ to $2.00 a$ in Figure $6 \mathrm{~b}$ show that the modes underwent a blue-shift as the depth of the PhC elements increased. The reason behind this shift can be explained by the increase of $n_{e f f}$ of the periodic structure, affecting its waveguiding properties. Additional information about the number and location of dual resonant modes can be extracted from the figure, and it can be seen that the waveguide accumulated two resonant modes in the vicinity of each other at shallow hole-depths of $d=0.30 a$ and $0.63 a$. Meanwhile, for larger values of $d$, the mode separation increased with an increase in the linewidth of individual modes.

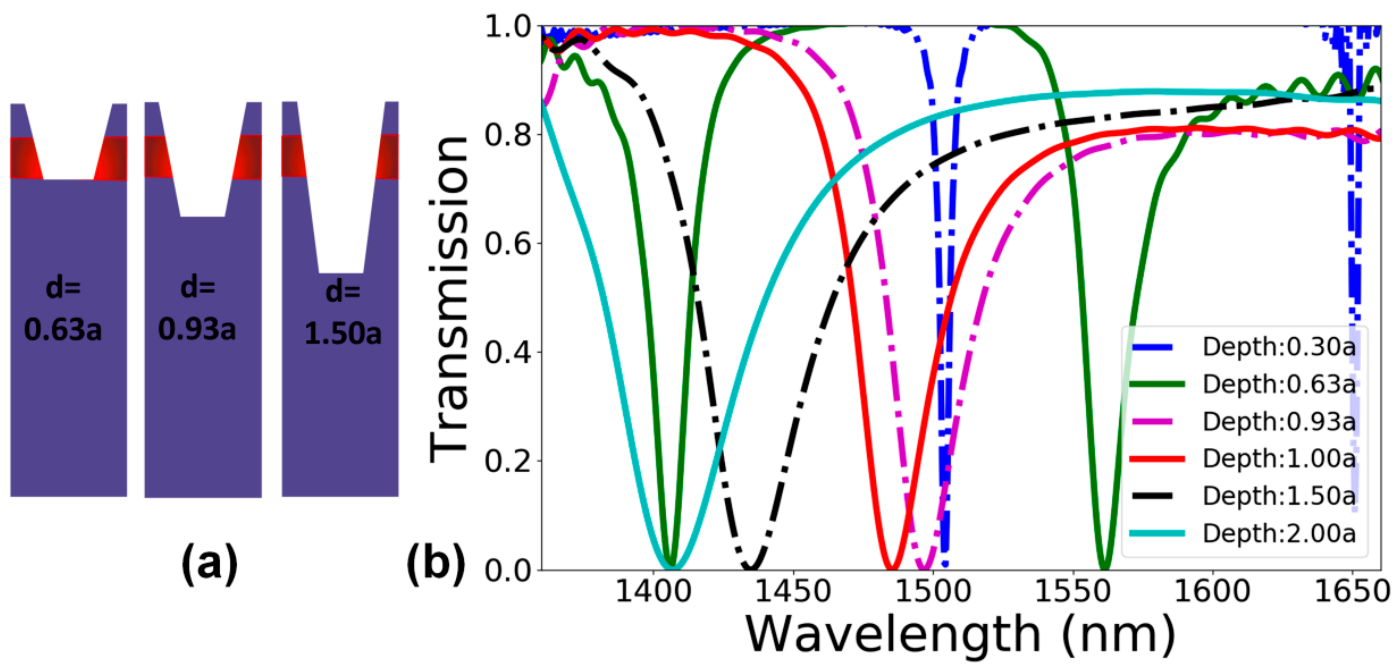

Figure 6. (a) PhC-elements with different values of $\mathrm{d}$ at $R_{t} / R_{b}=0.40 / 0.20 a$. (b) Transmission spectra of the PhC filter with variation in hole-depth value from $d=0.30 a$ to $2.00 a$ at $R_{t} / R_{b}=0.40 / 0.20 a$.

The performance considerations of the Fano-filter device, in terms of $\lambda_{\text {res }}$, linewidth, and QF for the hole-depth are shown in Figure 7. Figure 7a shows that the $\lambda_{\text {res }}$ of the considered resonant mode underwent a blue-shift in a slightly exponential manner in the 
range of 1400 to $1650 \mathrm{~nm}$ as the depth of the PhC elements increased. The linewidth of the modes (Figure $7 \mathrm{~b}$ ) increased in value, over a wide range from $4 \mathrm{~nm}$ to $60 \mathrm{~nm}$, as the depth increased. The QF of the modes (Figure 7c) had a very high value, in the range of 800 , for a sharp resonant mode appearing at $\lambda_{\text {res }}=1650 \mathrm{~nm}$ at a shallow depth of $d=0.30 a$. However, the QF suffered a huge drop, from 800 to 100 , at $\lambda_{\text {res }}=1560 \mathrm{~nm}$ as the depth of the air hole increased from $d=0.30 a$ to $0.63 a$. It is important to mention here that a depth of $=0.63 a$ means that the air holes are milled across the waveguide layer. The EM field distributions in the PhC structure during the occurrence of GMR for hole depths of $d=0.30 a$ and $1.5 a$ are shown in Figure $7 \mathrm{~d}$. The maxima and minima of the EM field are represented by red and blue, respectively. The resonant modes showed a strong coupling and field localization during GMR at the standard hole-depth of $d=0.93 a$. Meanwhile, at the depth of $d=1.5 a$, most of the field is transmitted and weak localization was observed in the upper portion of the waveguide.
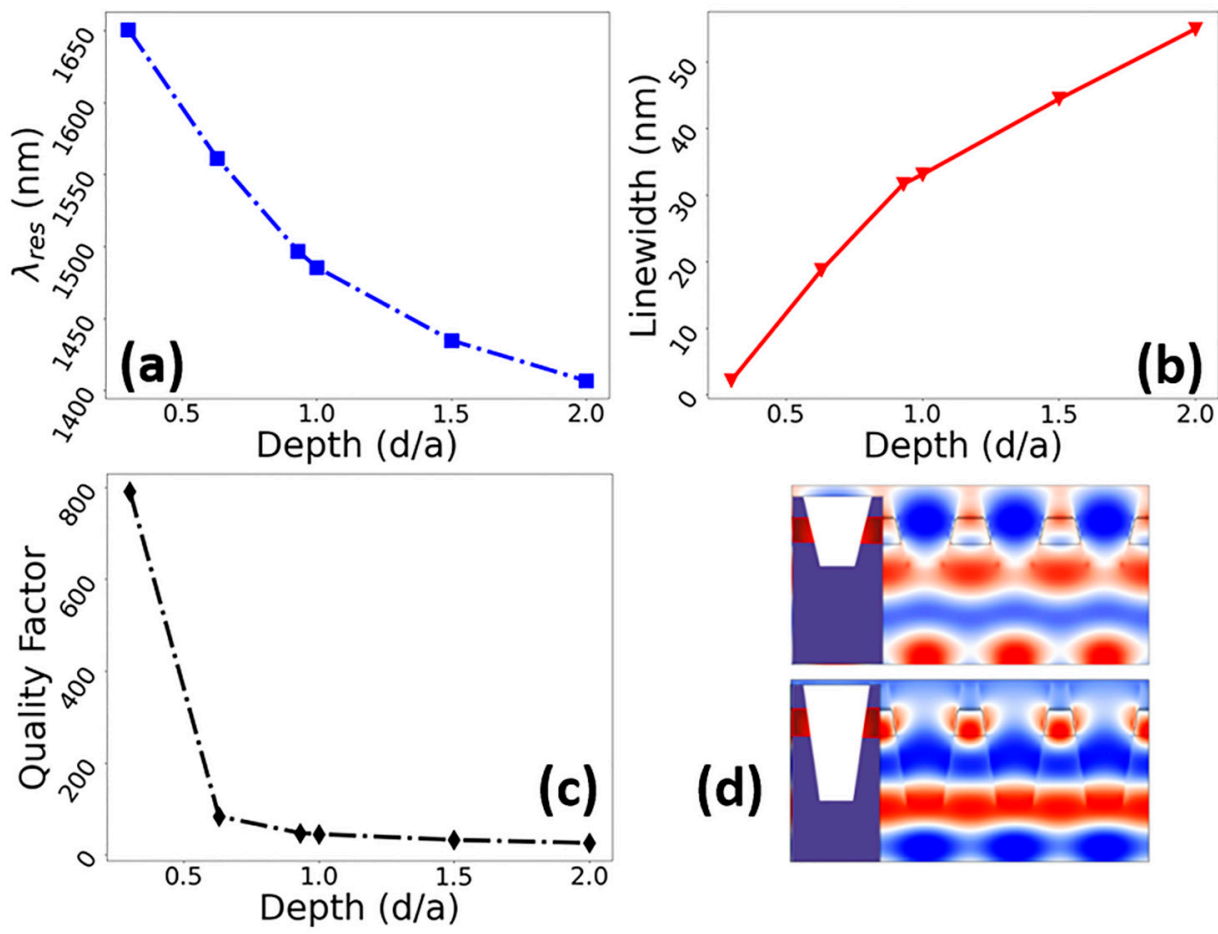

Figure 7. Spectral response of the Fano-filter for variation in the depth of the PhC-elements. (a) $\lambda_{\text {res }}$ as a function of $d$. (b) Linewidth as a function of $d$. (c) QF as a function of $d$. (d) EM field distribution for $d=0.93 a$ and $1.5 a$.

\subsection{Cladding-Layer Thickness Dependence of Resonances}

Besides the accumulation of surface charge, high-energy particle beams, such as those used in FIB technologies, can also have corrosive effects on the surface of the specimen. This corrosion mainly occurs during scanning of the surface for selection and focusing of the beam to the working area. This effect can be observed in the SEM images shown in Figure 2a,b, where the $10 \mathrm{~nm}$ Pt layer deposited on the surface was almost removed and the upper diameter of the milled holes seems to be rounded due to corrosion. An experimentally optimum hole shape of $R_{t} / R_{b}=0.4 / 0.20 a$ was selected for the numerical simulations, in order to investigate the effect of cladding layer thickness on the spectral properties of the PhC filter. The effect was studied in the absence of a cladding layer $c=0.00 a$, gradually increasing its thickness up to $c=0.50 a$ with a step size of $0.10 a$. The structural profiles of the numerical models for different values of the cladding layer are shown in Figure 8a. 


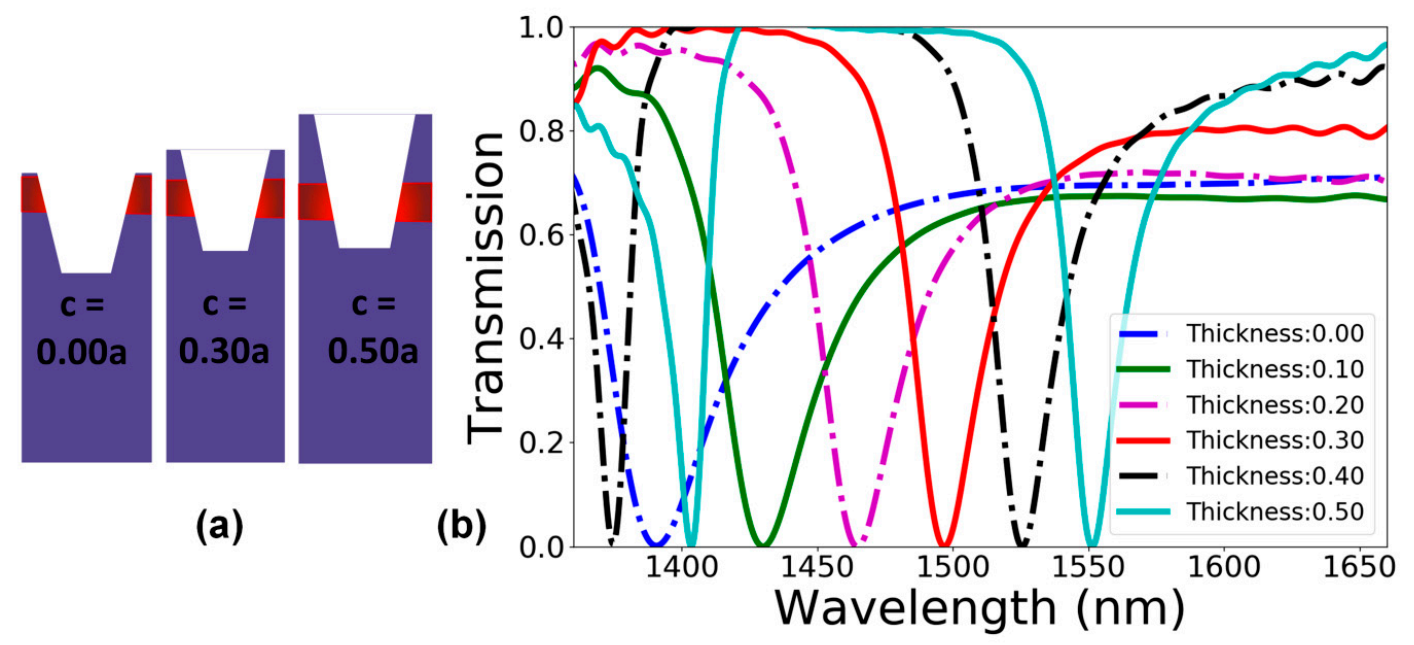

Figure 8. (a) Simulated dielectric PhC structures with conical shape and cladding thickness of $c=0.0 a, 0.30 a$ and $c=0.50 a$. (b) Transmission spectra for variation in cladding thickness values from $c=0.00 a$ to $0.50 a$.

The transmission spectra in Figure $8 \mathrm{~b}$ show that the resonant modes observed a redshift, along with a decrease in linewidth, as the thickness of the cladding layer increased. The appearance of a second resonant mode can also be observed in the vicinity of the main mode with thickness values of $c=0.40 a$ and $0.50 a$. Furthermore, $\lambda_{\text {res }}$ observed an almost linear red-shift (Figure 9a) in the range from $1390 \mathrm{~nm}$ to $1550 \mathrm{~nm}$ as the thickness of the cladding layer was increased. The linewidth of the resonant modes (Figure 9b) showed an overall decrease in value over a comparatively small range of values (from $48 \mathrm{~nm}$ to $20 \mathrm{~nm}$ ) as the cladding thickness increased. On the contrary, a rise in the QF (Figure 9c) could be observed with an increase in the value of $c$. The minimum $\mathrm{QF}$, for $c=0.00 a$, was observed to be 28, while the maximum achieved QF was in the range of 72 with $c=0.50 a$. The EM field distribution shown in Figure 9d depicts a weak localization of resonant modes for both extreme values of $c=0.00 a$ and $0.50 a$.

\subsection{Refractive Index Dependence of Resonances}

During thin-film deposition, the type of deposition technique used, as well as the operating parameters of the process, affect the quality and physical properties of the materials. These parameters may include the temperature inside the deposition chamber, vacuum conditions, plasma concentration, process recipe used, and ageing of the equipment. Considering the deposition technique, materials deposited using IBSD technology usually are of higher quality, compared to the use of the Chemical Vapor Deposition (CVD) technique. Taking into consideration the optical properties of the deposited materials, the variation in the refractive index must be considered, as it directly affects the spectral properties of the optical filter devices. To study this phenomenon, the refractive index of the waveguide layer was varied from $n_{2}=2.0$ to 2.5 , with a step size of 0.1 , in a standard numerical model with $R_{t} / R_{b}=0.30 / 0.30 a$ (Figure 10a).

The transmission spectra in Figure 10b show that the modes underwent a red-shift as the value of $n_{2}$ increased; while, at the same, the dual-mode properties of the waveguide became more obvious. The $\lambda_{\text {res }}$ plot in Figure 11a shows a perfectly linear red-shift in the range of $1400 \mathrm{~nm}$ to $1650 \mathrm{~nm}$ as $n_{2}$ increased. In contrast to the structural properties discussed in previous sections, the linewidth plot of the device in Figure 11b showed a linear increase in the range from 24 to $50 \mathrm{~nm}$ as the value of $n_{2}$ increased. As the $\lambda_{\text {res }}$ and linewidth values of the device increased, the QF of the device (Figure 11c for the considered resonant mode continuously decreased (from 60 to 34 ) as $n_{2}$ increased. Figure 11d depicts the EM field distribution in the periodic structure for $n_{2}=2.0$ and 2.5, showing weak and strong localization of resonant modes, respectively. The reason for this effect can be 
explained in terms of the contrast of the refractive index of the waveguide, with respect to its surrounding material. As the index contrast between the layers increases, the losses are reduced and confinement of the field inside the waveguide increases.
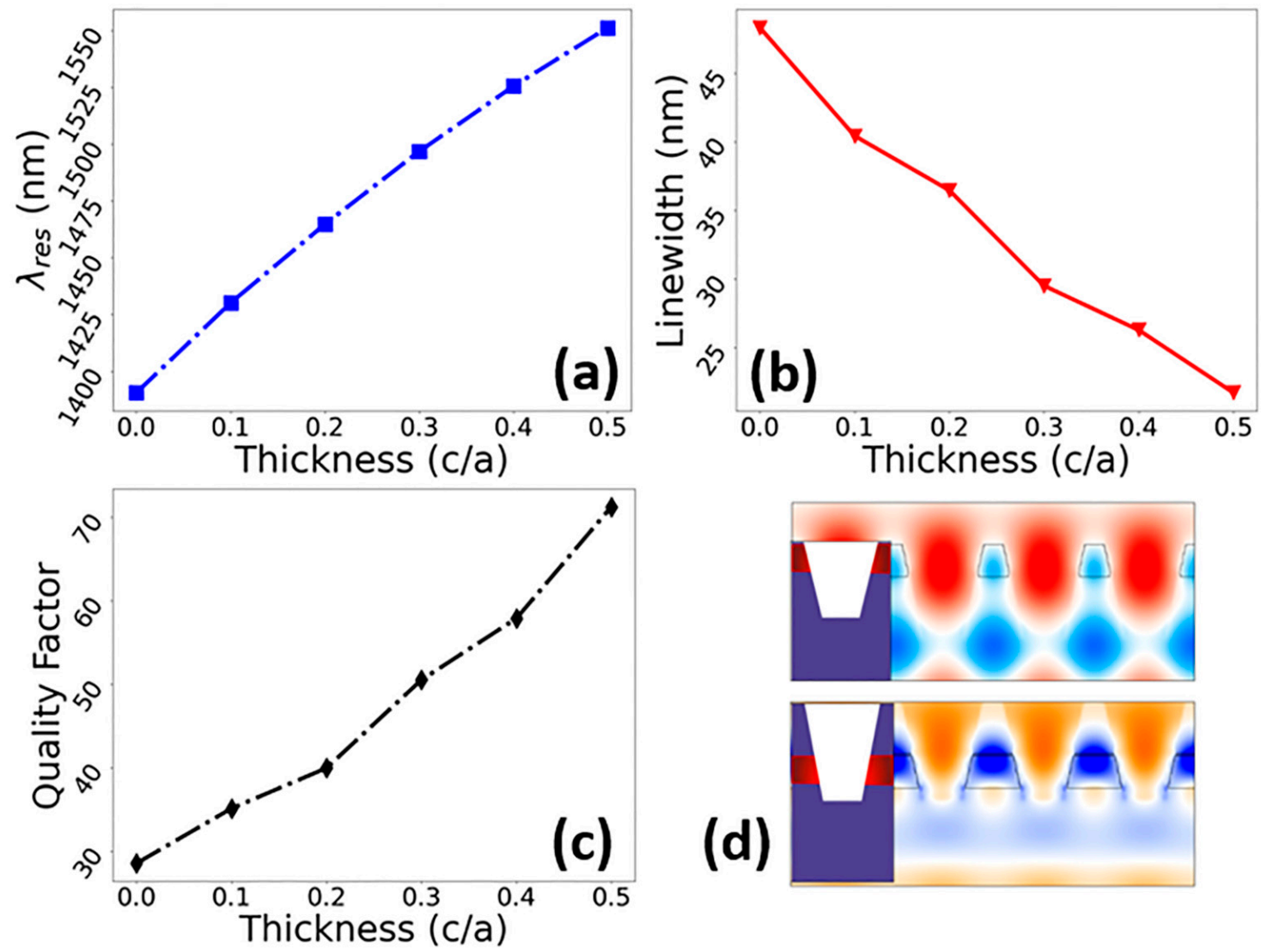

Figure 9. Spectral response of the Fano-filter for variation in the cladding layer thickness. (a) $\lambda_{\text {res }}$ as a function of $c$. (b) Linewidth as a function of $c$. (c) QF as a function of $c$. (d) EM field distribution for $c=0.00 a$ and $0.50 a$.

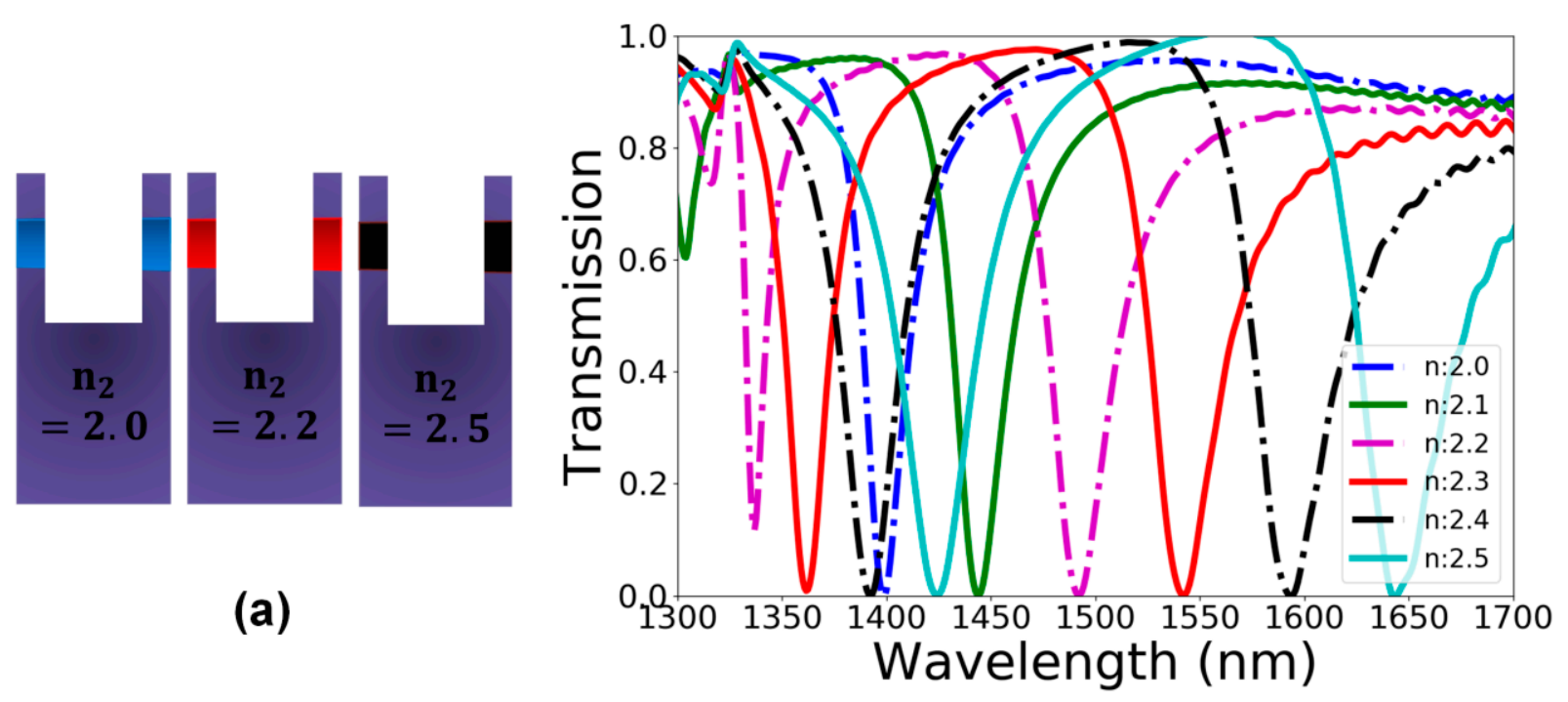

Figure 10. (a) Refractive index of the waveguide layer with $n_{2}=2.0,2.2$ and 2.5 depicted in different colors. (b) Transmission spectra for variation in the refractive index of the waveguide. 

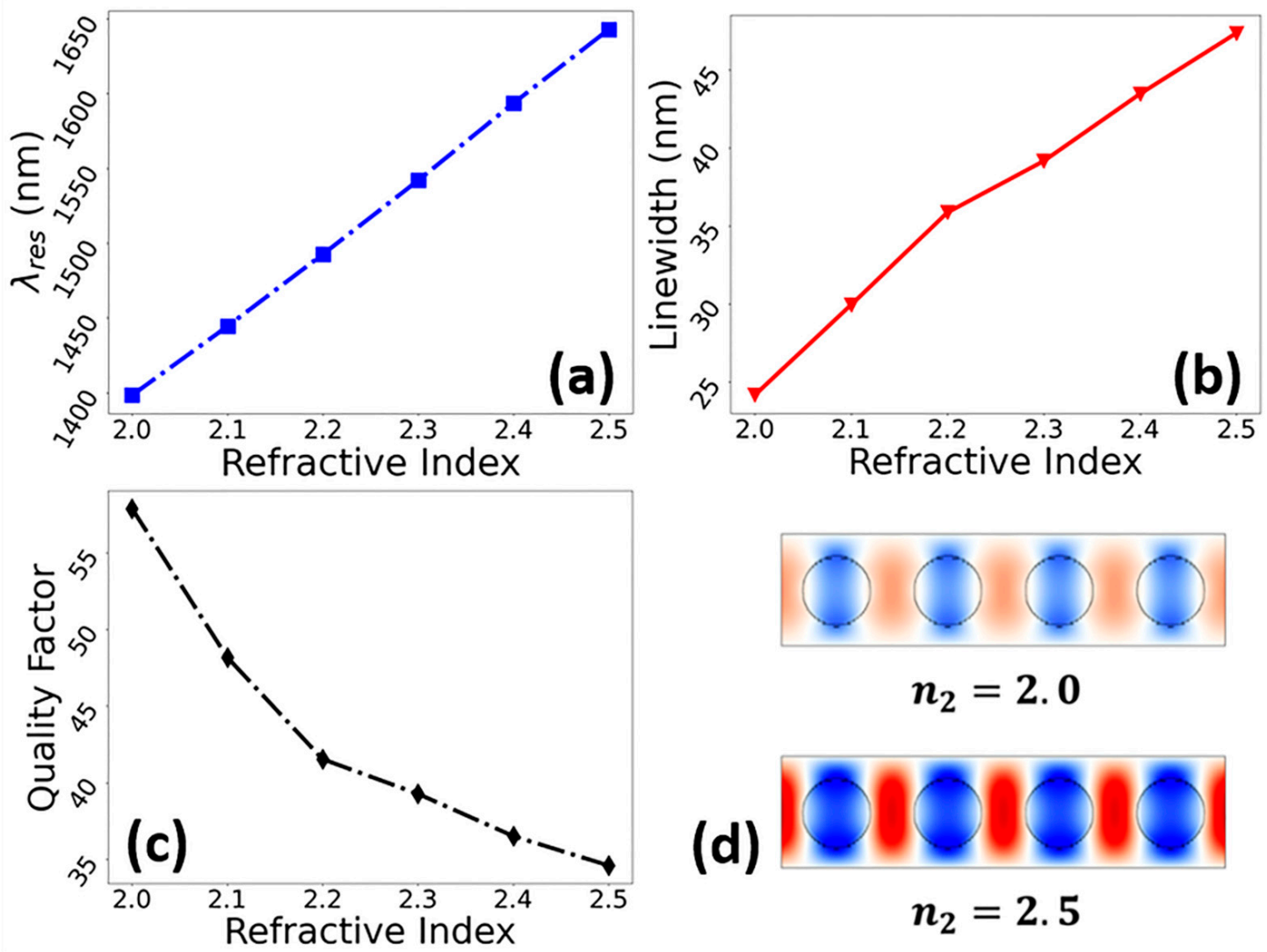

Figure 11. Spectral response of the Fano-filter for variation of refractive index of the waveguide layer. (a) $\lambda_{\text {res }}$ as a function of $n_{2}$. (b) Linewidth as a function of $n_{2}$. (c) QF as a function of $n_{2}$. (d) EM field distribution for $n_{2}=2.0$ and 2.5 .

\section{Conclusions}

In conclusion, a slab waveguide PhC-based Fano-filter was fabricated using low-cost dielectric materials, and its structural properties were analysed according to various FIB technology process parameters. The spectral characteristics were numerically investigated in accordance with the fabrication results. The standard theoretical model of the device has a PhC element shape of $R_{t} / R_{b}=0.30 / 0.30 a$, with a depth of $d=0.93 a$ and $c=0.30 a$. However, an optimum fabrication model was found, which had a PhC element shape of $R_{t} / R_{b}=0.40 / 0.20 a$, hole depth in the range of $d=0.93 a$ to $1.00 a$, and cladding thickness of $c=0.30 a$, corresponding to the ion-beam current of $300 \mathrm{pA}$ used during the milling process. The overall spectral quality of the Fano-filter was decreased when the shape and depth of the structures exceeded this mentioned range. The proposed Fano-filter was designed to operate in a spectral range around $\lambda_{\text {res }}=1500 \mathrm{~nm}$; however, the spectral properties of the fabricated device may vary in the range of 1400 to $1600 \mathrm{~nm}$ range, depending on the quality of the structures. The QF of the device was around 45, with minimum and maximum values of 35 and 800, respectively. Considering the physical properties, the $\lambda_{\text {res }}$ varied between 1400 to $1650 \mathrm{~nm}$ and the QF ranged from 34 to 58 when changing $n_{2}$ from 2.0 to 2.5. The simulation results can be further validated by comparison with the optical characterization results of the fabricated structures in the future. The device is proposed for use in optical filter-based applications in fibre optics communications, lasers, and sensors. 


\begin{abstract}
Author Contributions: Conceptualization, Y.K. and A.U.R.; methodology, Y.K., A.U.R., B.A.B. and M.N.; software, Y.K., A.U.R., B.A.B. and M.N.; validation, Y.K., A.U.R., B.A.B. and M.N.; formal analysis, Y.K., A.U.R., B.A.B. and M.N.; investigation, Y.K., A.U.R., B.A.B. and M.N.; resources, Y.K., A.U.R., B.A.B. and M.N.; data curation, Y.K., A.U.R., B.A.B. and M.N.; writing-original draft preparation, Y.K. and M.A.B.; writing-review and editing, Y.K., A.U.R., B.A.B., M.N. and M.A.B., S.N.K., N.L.K.; visualization, Y.K., A.U.R., B.A.B. and M.N.; supervision, Y.K. and M.A.B.; project administration, Y.K., N.L.K., S.N.K.; funding acquisition, M.A.B., N.L.K. and S.N.K. All authors have read and agreed to the published version of the manuscript.
\end{abstract}

Funding: This work was funded by the German Research Foundation (DFG) under the priority program SPP1327 to achieve sub-100-nm structures. And also the research was supported by the Ministry of Science and Higher Education of the Russian Federation in financing of new laboratories under the guidance of young scientists within the framework of the national Project "Science and Universities" (project FSSS-2021-0016) in the overview and comparative parts and under the FSRC "Crystallography and Photonics" of the Russian Academy of Sciences (the state task No. 007GZ/Ch3363/26) in the part of sensing devices.

Acknowledgments: The authors are grateful to all the members of the Nanophotonics group at the Institute of Nanostructure Technologies and Analytics (INA), University of Kassel, Germany and at BUITEMS Quetta for making this research possible.

Conflicts of Interest: The authors declare no conflict of interest.

\title{
References
}

1. John, S. Strong Localization of Photons in Certain Disordered Superlattices. Phys. Rev. Lett. 1987, 58, 2486. [CrossRef] [PubMed]

2. Yablonovitch, E. Inhibited Spontaneous Emission in Solid-State Physics and Electronics. Phys. Rev. Lett. 1987, 581-584. [CrossRef] [PubMed]

3. Magnusson, R.; Wang, S.S. New Principle for Optical Filters. Appl. Phys. Lett. 1992, 61, 1022-1024. [CrossRef]

4. Butt, M.A.; Khonina, S.N.; Kazanskiy, N.L. Recent Advances in Photonic Crystal Optical Devices: A Review. Opt. Laser Technol. 2021, 142, 107265. [CrossRef]

5. Fatemeh, G.; Entezar, S.R.; Razi, S. Graphene Based Photonic Crystal Optical Filter: Design and Exploration of the Tunability. Phys. Lett. A 2019, 383, 2551-2560.

6. Vlasov, Y.A.; O'boyle, M.; Hamann, H.F.; McNab, S.J. Active control of slow light on a chip with photonic crystal waveguides. Nature 2005, 438, 65-69. [CrossRef]

7. Wang, Q.; Xiao, M.; Liu, H.; Zhu, S.; Chan, C.T. Measurement of the Zak phase of photonic bands through the interface states of a metasurface/photonic crystal. Phys. Rev. B 2016, 93, 041415. [CrossRef]

8. Chen, F. A Tunable High-Efficiency Optical Switch Based on Graphene Coupled Photonic Crystals Structure. J. Mod. Opt. 2017, 64, 1531-1537. [CrossRef]

9. Fariborz, P.; Malmir, M.R. Reconfigurable All Optical Half Adder and Optical XOR and AND Logic Gates Based on 2D Photonic Crystals. Opt. Quantum Electron. 2020, 52, 1-8.

10. Baker, J.E.; Sriram, R.; Miller, B.L. Two-dimensional photonic crystals for sensitive microscale chemical and biochemical sensing. Lab Chip 2015, 15, 971-990. [CrossRef]

11. Butt, M.A.; Khonina, S.N.; Kazanskiy, N.L. 2D-Photonic Crystal Heterostructures for the Realization of Compact Photonic Devices Photonics Nanostruct.-Fundam. Appl. 2021, 44, 100903. [CrossRef]

12. Hadzialic, S.; Kim, S.; Sudbø, A.S.N.; Solgaard, O. Two-Dimensional Photonic Crystals Fabricated in Monolithic Single-Crystal Silicon. IEEE Photonics Technol. Lett. 2010, 22, 67-69. [CrossRef]

13. Khan, Y. Design and Numerical Simulation of Dielectric Photonic Crystal Devices and Investigation of an Optical Characterization Method. Ph.D. Thesis, University of Kassel, Kassel, Germany, 2017.

14. Chen, A.; Chua, S.-J.; Fonstad, C.G., Jr.; Wang, B.; Wilhelmi, O. Two-Dimensional Photonic Crystals Fabricated by Nanoimprint Lithography. Available online: https://www.researchgate.net/profile/Benzhong-Wang/publication/289980749_Patterning_of_ two-dimensional_photonic_crystal_structures_by_nanoimprint_lithography/links/5695fb3708ae3ad8e33d98a4/Patterning-oftwo-dimensional-photonic-crystal-structures-by-nanoimprint-lithography.pdf (accessed on 16 December 2021).

15. Huang, H.W.; Lin, C.H.; Huang, J.K.; Lee, K.Y.; Lin, C.F.; Yu, C.C.; Tsai, J.Y.; Hsueh, R.; Kuo, H.C.; Wang, S.C. Investigation of GaN-based Light Emitting Diodes with Nano-Hole Patterned Sapphire Substrate (NHPSS) by Nano-Imprint Lithography. Mater. Sci. Eng. B 2009, 164, 76-79. [CrossRef]

16. Götte, N.; Kusserow, T.; Winkler, T.; Sarpe, C.; Englert, L.; Otto, D.; Meinl, T.; Khan, Y.; Zielinski, B.; Senftleben, A.; et al. Temporally Shaped Femtosecond Laser Pulses for Creation of functional Sub-100 nm Structures in Dielectrics. In Optically Induced Nanostructures; König, K., Ostendorf, A., Eds.; de Gruyter: Berlin, Germany, 2015; pp. 47-72.

17. Rybin, M.V.; Khanikaev, A.B.; Inoue, M.; Samusev, K.B.; Steel, M.J.; Yushin, G.; Limonov, M.F. Fano resonance between Mie and Bragg scattering in photonic crystals. Phys. Rev. Lett. 2009, 103, 023901. [CrossRef] [PubMed] 
18. Fan, S.; Joannopoulos, J.D. Analysis of Guided Resonances in Photonic Crystal Slabs. Phys. Rev. B 2002, 65, 235112. [CrossRef]

19. Fan, S.; Suh, W.; Joannopoulos, J.D. Temporal coupled-mode theory for the Fano resonance in optical resonators. J. Opt. Soc. Am. A 2003, 20, 569-572. [CrossRef] [PubMed]

20. Limonov, M.F.; Rybin, M.V.; Poddubny, A.N.; Kivshar, Y.S. Fano Resonances in Photonics. Nat. Photonics 2017, 11, 543-554. [CrossRef]

21. Galli, M.; Portalupi, S.L.; Belotti, M.; Andreani, L.C.; O’Faolain, L.; Krauss, T.F. Light scattering and Fano resonances in high-Q photonic crystal nanocavities. Appl. Phys. Lett. 2009, 94, 071101. [CrossRef]

22. Sturmberg, B.C.; Dossou, K.B.; Botten, L.C.; McPhedran, R.C.; De Sterke, C.M. Fano resonances of dielectric gratings: Symmetries a broadband filtering. Opt. Express 2015, 23, A1672-A1686. [CrossRef]

23. Shuai, Y.; Zhao, D.; Singh Chadha, A.; Seo, J.H.; Yang, H.; Fan, S.; Ma, Z.; Zhou, W. Coupled double-layer Fano resonance photonic crystal filters with lattice-displacement. Appl. Phys. Lett. 2013, 103, 241106. [CrossRef]

24. Cao, G.; Dong, S.; Zhou, L.M.; Zhang, Q.; Deng, Y.; Wang, C.; Zhang, H.; Chen, Y.; Qiu, C.W.; Liu, X. Fano resonance in artificial photonic molecules. Adv. Opt. Mater. 2020, 8, 1902153. [CrossRef]

25. Nguyen, V.A.; Ngo, Q.M.; Le, K.Q. Efficient color filters based on Fano-like guided-mode resonances in photonic crystal slabs. IEEE Photonics J. 2018, 10, 1-8. [CrossRef]

26. Caselli, N.; Intonti, F.; La China, F.; Riboli, F.; Gerardino, A.; Bao, W.; Bargioni, A.W.; Li, L.; Linfield, E.H.; Pagliano, F.; et al. Ultra-subwavelength phase-sensitive Fano-imaging of localized photonic modes. Light Sci. Appl. 2015, 4, e326. [CrossRef]

27. Shuai, Y.; Zhao, D.; Tian, Z.; Seo, J.H.; Plant, D.V.; Ma, Z.; Fan, S.; Zhou, W. Double-layer Fano resonance photonic crystal filters. Opt. Express 2013, 21, 24582-24589. [CrossRef]

28. Hossain, M.N.; Justice, J.; Lovera, P.; McCarthy, B.; O’Riordan, A.; Corbett, B. High aspect ratio nano-fabrication of photonic crystal structures on glass wafers using chrome as hard mask. Nanotechnology 2014, 25, 355301. [CrossRef] [PubMed]

29. Joshi-Imre, A.; Bauerdick, S. Direct-Write Ion Beam Lithography. J. Nanotechnol. 2014, 2014, 170415. [CrossRef]

30. Steve, R.; Robert, P. A Review of Focused Ion Beam Applications in Microsystem Technology. J. Micromech. Microeng. 2001, 11, 287-300.

31. Lonergan, A.; Breda, M.; O'Dwyer, C. Metallo-Dielectric Photonic Crystals and Bandgap Blue-Shift. arXiv 2021, arXiv:2105.06280.

32. Prather, D.W.; Shi, S.; Sharkawy, A.; Murakowski, J.; Schneider, G.J. Photonic Crystals, Theory, Applications and Fabrications; Wiley: Hoboken, NJ, USA, 2009.

33. Ullah, A.; Wilke, H.; Memon, I.; Shen, Y.; Nguyen, D.T.; Woidt, C.; Hillmer, H. Stress relaxation in dual ion beam sputtered $\mathrm{Nb}_{2} \mathrm{O}_{5}$ and $\mathrm{SiO}_{2}$ thin films: Application in a Fabry-Pérot filter array with 3D nanoimprinted cavities. J. Micromech. Microeng. 2015, 25, 055019. [CrossRef]

34. Lambert, E.; Fiers, M.; Nizamov, S.; Tassaert, M.; Johnson, S.G.; Bienstman, P.; Bogaerts, W. Python Bindings for the Open-Source Electromagnetic Simulator Meep. Comput. Sci. Eng. 2010, 13, 53-65. [CrossRef]

35. Oskooi, A.F.; Roundy, D.; Ibanescu, M.; Bermel, P.; Joannopoulos, J.D.; Johnson, S.G. Meep: A Flexible Free-Software Package for Electromagnetic Simulations by the FDTD Method. Comput. Phys. Commun. 2010, 181, 687-702. [CrossRef] 